\title{
Evaluation of a simplified vitamin supplement inventory developed for the Women's Health Initiative
}

\author{
Ruth E Patterson*, Lisa Levy, Lesley Fels Tinker and Alan R Kristal \\ Fred Hutchinson Cancer Research Center, 1100 Fairview Avenue North, Seattle, WA 98109, USA
}

Submitted 11 August 1998: Accepted 22 January 1999

\begin{abstract}
Objective: To evaluate the accuracy of a simplified inventory procedure for assessing nutrient intake from vitamin and mineral supplements.

Design: Participants brought their supplements to a clinic. An interviewer conducted the supplement inventory procedure, which consisted of recording data on the type of multiple vitamin and single supplements used. For the multiple vitamins, the interviewer recorded the exact dose for a subset of nutrients (vitamin C, calcium, selenium). For other nutrients, we imputed the dose in multiple vitamins. The dose of all single supplements was recorded. Labels of the supplements were photocopied and we transcribed the exact nutrient label data for the criterion measure. Spearman correlation coefficients were used to assess precision of nutrient intakes from the simplified inventory compared to the criterion measure.

Setting/subjects: Data are from 104 adult vitamin supplement users in Washington state.

Results: Correlation coefficients between nutrient intake estimated from the simplified inventory compared to the criterion measure were high $(0.8-1.0)$ for those nutrients (vitamin C, calcium, selenium) for which the interviewer recorded the exact dose contained in multiple vitamins. However, for nutrients for which imputations were made regarding dose in multiple vitamins, correlation coefficients ranged from good (0.8 for vitamin E) to poor (0.3 for iron).

Conclusions: The simplified inventory is rapid ( $4-5 \mathrm{~min}$ ) and practical for large-scale studies. The precision of nutrient estimates using this procedure was variable, although excellent for the subset of nutrients for which the dose was recorded exactly. This study illustrates many of the challenges of collecting high quality supplement data.
\end{abstract}

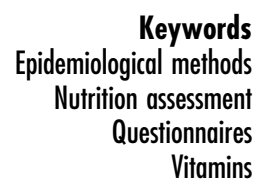

Vitamin and mineral supplements have been associated with decreased risk of some cancers ${ }^{1}$, cardiovascular disease $^{2}$ and other chronic diseases ${ }^{3}$. In addition, nutrients from supplements can provide a large proportion of total nutrient intake in the diet ${ }^{4}$. Therefore, accurate information on supplement use is needed to examine the role of diet in chronic disease ${ }^{5}$.

Assessing supplement use presents many difficulties. A brand-name inventory is not feasible for many studies because there are more than 3400 vitamin and mineral preparations available to consumers ${ }^{6}$ and we know of no readily accessible, well-maintained supplement database. Since many multiple vitamins contain over 30 nutrients, abstracting all the nutrient data from the labels of supplement bottles is a timeconsuming and error-prone task. Alternatively, investigators can choose to abstract label data about only a few nutrients of primary interest to the study. However, for aetiological research or for research in which baseline data are collected for future outcome analysis, it is usually unwise and poor economy to restrict data collection to a limited set of nutrients ${ }^{7}$. If investigators only collect data about one or two nutrients, they forego all ability to reanalyse data for nutrients that may become of interest in the future.

In response to the difficulties outlined above, we developed a simplified vitamin supplement inventory procedure for a large multicentre study of chronic disease in post-menopausal women: the Women's Health Initiative ${ }^{8,9}$. This simplified inventory captures information on the major classes of multiple vitamins, less common mixed supplements, and all single supplements. This assessment procedure was designed for non-nutritionist interviewers.

The objective of this study was to evaluate the accuracy of this simplified vitamin inventory procedure for assessing supplement use. Specifically, we examined the bias and precision of estimating supplemental vitamin and mineral intakes from the simplified inventory compared to a more accurate but burdensome 
measure: intake determined from supplement label values.

\section{Methods}

\section{Study participants}

This investigation was an ancillary study to the 19951996 Washington State Cancer Risk Behavior Survey, a random digit dial survey of adults, 18 years of age and older, designed to monitor attitudes and behaviour related to cancer risk and prevention ${ }^{10}$. Residents were eligible for this ancillary study if they used vitamin or mineral supplements at least three times per week and lived close to the Fred Hutchinson Cancer Research Center in Seattle, Washington. We excluded people taking only herbal supplements and women taking prenatal vitamins.

\section{Study procedures}

Approximately 1-2 months after completion of the statewide survey, we attempted to reach potential participants by telephone to schedule a clinic appointment. At the clinic visit, we completed the supplement inventory and photocopied the labels of the participants' supplements. Of 192 eligible supplement users, 104 (54.2\%) completed the ancillary study, 24 (12.5\%) could not be contacted and $64(33.3 \%)$ refused to participate or did not complete the study.

\section{Simplified supplement inventory}

To enable non-nutritionists to collect the supplement data in a standardized manner, the first page of the form contained definitions and common examples of the multiple vitamin classes. During the clinic interview, the interviewer (who was not a nutritionist) examined the participants' supplement bottles and recorded information on use of:

1. three classes of multiple vitamins (one-a-day type without minerals, one-a-day type with minerals and stress supplements);

2. all other mixtures (e.g. B-complex, calcium plus magnesium);

3. all single supplements (pills containing a single vitamin or mineral).

The interviewer recorded exact doses of a subset of nutrients of special interest contained in multiple vitamins (vitamin C, calcium and selenium) in addition to the doses of nutrients contained in other mixtures and in single supplements. For each supplement, the interviewer queried the participant about the frequency (times per week) and duration of use (months or years used).

\section{Criterion measure: intake determined from supplement label values}

After completing the supplement inventory, the interviewer photocopied the labels on the participants' supplement bottles. To obtain information on supplement use over the past year, we used a scripted, openended interview with questions on usual use per week with probes for changes in routines (e.g. vacation or illness).

At a later date, the interviewer transcribed dose information on all nutrients contained in the supplement(s) onto a standardized form. We compared the final dataset to the photocopied supplement labels to ensure the accuracy of the criterion measure.

\section{Analysis}

We calculated average daily dose of nutrients over the past year for the simplified inventory and the criterion measure by summing across multiple vitamins and single supplements after adjusting for frequency and duration of use. We wrote computer algorithms for nutrient conversions. We calculated these variables for the nutrients most frequently taken as single supplements (vitamin C, vitamin E and calcium) and for a set of nutrients of scientific interest (folic acid, iron and selenium). Assumptions for the formulations of the three classes of multiple vitamins were as follows: (i) one-a-day multivitamins without minerals: $30 \mathrm{mg}$ $\alpha \mathrm{TE}$ ( $\alpha$ tocopherol equivalents) vitamin $\mathrm{E}$ and $400 \mu \mathrm{g}$ folic acid; (ii) one-a-day multivitamins with minerals: vitamins as above plus $18 \mathrm{mg}$ iron; (iii) stress multivitamins: $500 \mathrm{mg}$ vitamin $\mathrm{C}, 30 \mathrm{mg} \alpha \mathrm{TE}$ vitamin $\mathrm{E}$ and $400 \mu \mathrm{g}$ folic acid. These assumptions were based on the composition of leading brands ${ }^{11}$, characteristics of supplement products in the USA ${ }^{5}$, and informal surveys of vitamin supplements available at drug stores in King County, Washington.

To assess bias we determined the per cent of respondents taking supplemental vitamins and minerals, and the mean and median intake among users. Data on nutrients from supplements were highly skewed and non-normal regardless of data transformation. Therefore, Spearman correlation coefficients were used to assess precision of nutrient intakes from the simplified inventory compared to the criterion measure.

\section{Results}

Study participants $(n=104)$ had a mean age of 44 (12.5 SD) years, 57\% were women, 91\% Caucasian and $54 \%$ had a college education. The per cent of participants obtaining a nutrient from any supplement was quite similar for the simplified inventory and the criterion measure (supplement intakes determined from the label), with the exception of iron (Table 1). About $8 \%$ more participants took iron than was 
Table 1 Comparison of daily supplemental nutrient intakes (summed across all supplements) estimated from a simplified supplement inventory procedure and determined from supplement label values (the criterion measure) $(n=104)$

\begin{tabular}{|c|c|c|c|c|c|c|}
\hline \multirow[b]{2}{*}{ Nutrient } & \multicolumn{3}{|c|}{$\begin{array}{l}\text { Supplement intake estimated } \\
\text { from a simplified supplement } \\
\text { inventory procedure }\end{array}$} & \multicolumn{3}{|c|}{$\begin{array}{l}\text { Supplement intake determined } \\
\text { from label values }\end{array}$} \\
\hline & Users* (\%) & Mean† (SD) & Median† & Users (\%)* & Mean† (SD) & Median† \\
\hline Vitamin C (mg)‡ & 92.3 & 597 (938) & 380 & 95.2 & $643(1048)$ & 413 \\
\hline Calcium (mg) $\ddagger$ & 74.0 & 308 (317) & 174 & 76.9 & $370(415)$ & 207 \\
\hline Selenium $(\mu \mathrm{g}) \ddagger$ & 60.6 & $35(57)$ & 20 & 62.5 & $41(82)$ & 20 \\
\hline Vitamin $\mathrm{E}(\alpha \mathrm{TE}) \S$ & 89.4 & $193(296)$ & 47 & 90.4 & $220(299)$ & 86 \\
\hline Folic acid $(\mu \mathrm{g}) \S$ & 84.6 & 435 (331) & 400 & 84.6 & $360(246)$ & 380 \\
\hline Iron $(\mathrm{mg}) \S$ & 66.3 & $23(21)$ & 18 & 74.0 & $17(17)$ & 17 \\
\hline
\end{tabular}

* Participants taking any supplement(s) containing the nutrient.

† Intake among participants taking any supplement(s) containing the nutrient.

$\ddagger$ For the simplified inventory, we recorded the exact dose of the nutrient if taken in multiple vitamins.

$\S$ For the simplified inventory, we imputed the dose of the nutrient if taken in multiple vitamins.

estimated from the simplified inventory. There was little evidence for bias, as the mean and median nutrient intakes estimated using the supplement inventory procedure were very similar to the criterion measure.

Correlation coefficients between nutrient intake estimated using the simplified inventory compared to the criterion measure were uniformly high $(0.8-1.0)$ for those nutrients (vitamin C, calcium and selenium) for which interviewers recorded exact doses contained in multiple vitamins (Table 2). However, for nutrients for which assumptions were made regarding the formulation of multiple vitamins, correlation coefficients ranged from good ( 0.8 for vitamin $\mathrm{E}$ ) to poor ( 0.3 for iron).

\section{Discussion}

We estimate that the simplified supplement inventory generally took $4-5 \mathrm{~min}$ per respondent and, therefore, is practical for in-person interviews. The simplified inventory allows an investigator to collect fairly precise data about a limited set of nutrients of primary interest

Table 2 Correlations between supplemental nutrient intakes (summed across all supplements) estimated from a simplified supplement inventory and determined from supplement label values (the criterion measure) $(n=104)$

\begin{tabular}{lccc}
\hline Nutrient & $\begin{array}{c}\text { Users* } \\
(\%)\end{array}$ & $\begin{array}{c}\text { Correlation } \\
\text { coefficient† }\end{array}$ & $\begin{array}{c}95 \% \\
\text { confidence } \\
\text { interval }\end{array}$ \\
\hline Vitamin C $\ddagger$ & 95.2 & 0.89 & $0.83-0.92$ \\
Calcium $\ddagger$ & 76.9 & 0.76 & $0.65-0.84$ \\
Selenium $\ddagger$ & 62.5 & 0.96 & $0.94-0.98$ \\
Vitamin E§ & 90.4 & 0.84 & $0.77-0.89$ \\
Folic acid§ & 84.6 & 0.61 & $0.45-0.73$ \\
Iron§ & 74.0 & 0.29 & $0.07-0.49$
\end{tabular}

* Participants taking any supplement(s) containing the nutrient.

† Spearman correlation coefficients among supplement users.

$\ddagger$ For the simplified inventory, we recorded the exact dose of the nutrient if taken in multiple vitamins

$\S$ For the simplified inventory, we imputed the dose of the nutrient if taken in multiple vitamins. by abstracting label information on the nutrient doses in multiple vitamins as well as intake from single supplements. However, the inventory also captures data on the types of multiple vitamin used such that investigators can analyse data for other nutrients by imputing the doses in multiple vitamins. In this study, the nutrient imputations were quite accurate for vitamin $\mathrm{E}$ and folic acid, but were less successful for iron because iron was present in multiple vitamin formulations that typically do not contain iron (e.g. stress multiple vitamins). In addition, there was substantial variability in the dose of iron in multiple vitamins.

Besides the limitation of the simplified inventory itself, another source of error in assessing supplemental nutrient intake can arise from errors by the interviewers. Interviewers may incorrectly classify the different types of multiple vitamins or make mistakes or omissions when recording doses, frequency or duration of use. In addition, some labels are confusing or unclear as to the dose of the nutrients. For example, the label may provide information on the amount of compound (e.g. mg of calcium carbonate) rather than the amount of nutrient (e.g. elemental calcium). Other supplement formations have split doses (e.g. six pills per day), which requires calculation of the total daily dose. In addition, different units of dose (e.g. RE vs. $\mathrm{mg}$ ) for the same nutrients necessitates the use of conversion factors. These errors may be reduced by employing nutritionists; however this may not be feasible in many study settings.

We computerized the simplified inventory for use in the Women's Health Initiative. Trained non-nutritionists conducted the inventory at a computer station and directly entered data about multiple vitamin(s) and single supplement(s): dose, frequency and duration of use. The program consists of a series of screens that prompts coders to enter information on the supplements, allows them to enter the dose in any unit used on the label, and incorporates range checks for quality assurance. 
The simplified inventory can be easily modified to collect precise data about any supplemental nutrient of central importance to a study. For example, we modified this form for the Olestra Post-Marketing Surveillance Study, which is a large observational study on the effect of olestra consumption on serum concentrations of fat-soluble vitamins and carotenoids ${ }^{12}$. Specifically, we modified the vitamin assessment inventory to collect exact dose data on vitamins $\mathrm{A}, \mathrm{D}, \mathrm{E}, \mathrm{K}$ and $\beta$-carotene in multiple vitamins in addition to single supplements.

For investigations as a whole, the objective of exposure measurement is to obtain measurements that are the minimum necessary to meet the research objective $^{13}$. Unfortunately, accuracy and practicality of data collection methods are often inversely correlated. Our data indicated that a simplified vitamin inventory was practical for large-scale research studies. The precision of nutrient estimates using this procedure was variable, although excellent for the subset of nutrients for which the dose was recorded exactly. This study illustrates many of the challenges involved in assessing nutrient intake from vitamin and mineral supplements.

\section{Acknowledgements}

This research was supported by P01 CA 34847 and R01 CA 64138 from the National Cancer Institute.

\section{References}

1 Patterson RE, White E, Kristal AR, Neuhouser ML, Potter JD. Vitamin supplements and cancer risk: a review of the epidemiologic evidence. Cancer Causes Control 1997; 8: 786-802.

2 Losonczy KG, Harris TB, Havlik RJ. Vitamin E and vitamin C supplement use and risk of all-cause and coronary heart disease mortality in older persons: the Established Populations for Epidemiologic Studies of the Elderly. Am. J. Clin. Nutr. 1996; 64: 190-6.

3 Reid IR, Ames RW, Evans MC, Gamble GD, Sharpe SJ. Effect of calcium supplementation on bone loss in postmenopausal women. N. Engl. J. Med. 1993; 328: 460-4.

4 Patterson RE, Kristal AR, Carter RA, Fels-Tinker L, Bolton MP, Agurs-Collins T. Measurement characteristics of the Women's Health Initiative food frequency questionnaire. Ann. Epidemiol. 1999; 9: 178-97.

5 Block G, Sinha R, Gridley G. Collection of dietary supplement data and implications for analysis. Am. J. Clin. Nutr. 1994; 59 (Suppl.): S232-9.

6 Park YK, Kim I, Yetley EA. Characteristics of vitamin and mineral supplement products in the United States. Am.J. Clin. Nutr. 1991; 54: 750-9.

7 Block G, Hartman AM. Dietary assessment methods. In: Moon TE, Micozzi MS, eds. Nutrition and Cancer Prevention. Investigating the Role of Micronutrients. New York: Marcel Dekker, 1989: 159-60.

8 Rossouw JE, Finnegan LP, Harlan WR, Pinn VW, Clifford C, McGowan JA. The evolution of the Women's Health Initiative: perspectives from the NIH. J. Am. Med. Womens Assoc. 1995; 50(2): 50-5.

9 Tinker LF, Burrows ER, Henry H, Patterson RE, Rupp J, Van Horn L. The Women's Health Initiative: overview of the nutrition components. In: Kris Etherton P, Krummel D, eds. Nutrition and Women's Health. Frederick, MD: Aspen Publishers, 1996: 510-42.

10 Kristal AR, Levy L, Patterson RE, Li SS, White E. Trends in food label use associated with new nutrition labeling regulations. Am.J. Public Health 1998; 88: 1212-15.

11 Physicians' Desk Reference for Nonprescription Drugs, 17th edn. Montvale, NJ: Medical Economics Data Publication Co., 1996.

12 Kristal AR, Patterson RE, Neuhouser ML, et al. The Olestra Post-Marketing Surveillance Study: design and baseline results from the sentinel site. J. Am. Diet. Assoc. 1998; 98 1290-6.

13 Armstrong BK, White E, Saracci R. Principles of Exposure Measurement in Epidemiology. Monographs in Epidemiology and Biostatistics, Vol. 21. Oxford: Oxford University Press, 1994. 